\title{
Polymerase chain reaction for detection of herpes simplex virus encephalitis
}

\author{
J Aslanzadeh, D J Skiest
}

\begin{abstract}
A patient with herpes simplex virus encephalitis is described. The polymerase chain reaction (PCR) was used to confirm the diagnosis in cerebrospinal fluid. PCR allows the rapid diagnosis of many infectious organisms, such as HSV, in which prompt diagnosis is essential.
\end{abstract}

(F Clin Pathol 1994;47:554-555)

Herpes simplex virus (HSV) encephalitis is rare but frequently fatal. Recent reports on the efficacy of antiviral agents, such as acyclovir, administered early in the infection have made its rapid diagnosis extremely important. The polymerase chain reaction (PCR) is a widely used and powerful technique to detect the presence of an infectious agent in clinical specimens. ${ }^{1}$ The method involves primer mediated, enzymatic amplification of the specific DNA sequence. The technique has been shown to be effective in detecting HSV in cerebrospinal fluid specimens from patients with documented HSVE as early as 24 hours after the onset of clinical symptoms. ${ }^{2}$ We report the use of PCR for detecting HSV DNA in the cerebrospinal fluid from a patient who presented with symptoms. Computed axial tomography scanning (CT) and magnetic resonance imaging (MRI) findings were compatible with HSV encephalitis.

\section{Case report}

A 79 year old white woman with a history of complex partial seizures controlled by carbamazepine was admitted to hospital in December. She had been confused, had unsteady gait, and dizziness for three days. She denied headache, loss of consciousness, focal weakness, neck stiffness, fever, or chills. Her medical history included pemphigus vulgaris in addition to partial complex seizures. She resided in Connecticut and had not travelled recently.

Physical examination was compatible with an awake but confused elderly woman. Her temperature was $38.3^{\circ} \mathrm{C}$. The relevant clinical findings on examination included bibasilar lung crackles and abnormal neurological signs, characterised by confusion, mild leftsided weakness, and a wide based gait. An electroencephalogram was performed. This indicated abnormal spikes over the right temporal lobe. An initial CT scan of the head was normal except for generalised cortical atrophy; but a repeat scan one week later revealed a low attenuation signal within the right temporal lobe. An MRI scan of the head showed an increase in signal in the right insular cortex, extending into the parieto-temporal area and several areas of abnormal white matter activity. The peripheral white cell count was $5 \cdot 2 \times 10^{9} / 1$. A lumbar puncture was performed, with the results as follows: $48 \times 10^{6} / 1$ white cells (differential 98\% mononuclear cells, $2 \%$ neutrophils), $153 \times$ $10^{6} / 1 \mathrm{red}$ blood cells, glucose $4.6 \mathrm{mmol} / 1$, protein $790 \mathrm{~g} / 1$, VDRL was non-reactive, and cryptococcal antigen by latex agglutination was negative. The cerebrospinal fluid Gram stain and acid fast stains were negative, and bacterial and mycobacterial cultures were also negative.

The initial cerebrospinal fluid sample was negative for antibodies to $\mathrm{HSV}$, while a second sample obtained three weeks later had a titre of $1 / 40$. The HSV antibody titre was determined using an indirect fluorescence antibody (IFA) test according to the manufacturer's protocol (Gull laboratories Inc. Salt Lake City, Utah, USA). Briefly, slides containing reaction sites, precoated with HSV infected cells, were overlaid with $20 \mu \mathrm{l}$ of cerebrospinal fluid and incubated at room temperature for 30 minutes. Slides were then washed with phosphate buffered saline (PBS) and the reaction sites were covered with $20 \mu$ l of fluorescein conjugated goat antihuman immunoglobulin. After 30 minutes of incubation at room temperature slides were washed with PBS and viewed under a fluorescence microscope. To determine whether HSV antibody was synthesised intrathecally, rubella antibody titre was also determined on all cerebrospinal fluid samples using the Rubascan latex agglutination test (Becton Dickinson, Cokeysville, Maryland, USA). Both cerebrospinal fluid samples from our patient contained no rubella antibodies, indicating that the HSV antibody was intrathecally synthesised and that there was no loss of blood-brain barrier.

PCR was carried out for 35 cycles with a set of primers that bracketed a 92 base pair segment unique to the HSV DNA polymerase gene (primer 1-5'-CATCACGACCCGGAGAGGGAC-3', primer 2-5'-GGGCCAGGCGCTTGTTGGTGTA-3', and oligonucleotide probe 5'-GTCCTCACCGCCGAACTGAG-3'). ${ }^{3}$ The primers and PCR protocol used in this report have known 


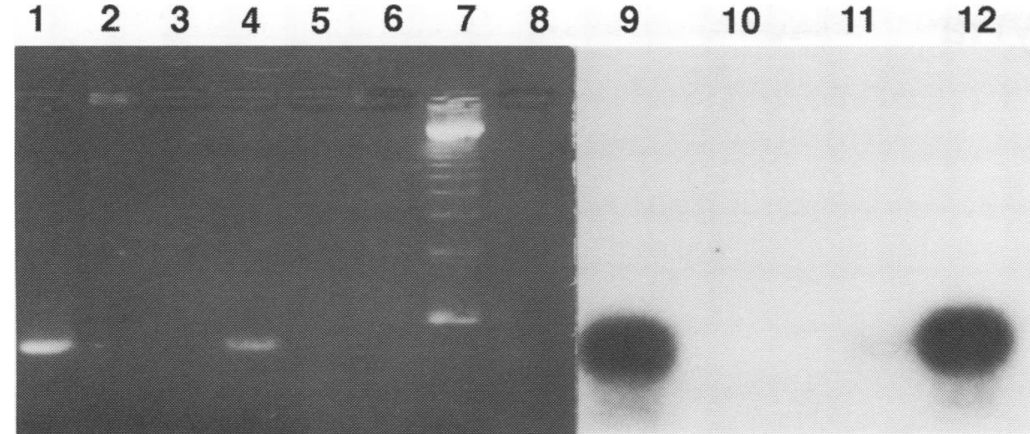

Composite prints of agarose gel electrophoresis (lane 1 positive control, lanes 2, 3 negative controls, lane 4 cerebrospinal fluid from a patient with $H S V$ encephalitis, lanes 5, 6, 8 blank wells, and lane 7123 base pair ladder molecular weight standard) and Southern blot analysis (lane 9 positive control, lanes 10, 11 negative controls, lane 12 cerebrospinal fluid from a patient with HSV encephalitis) of HSV DNA amplified by PCR.

sensitivity and specificity to detect less than 10 virus particles. ${ }^{3}$ Results from our patient show that PCR was positive on the initial cerebrospinal fluid sample, while a repeat PCR on the second sample obtained three weeks later, following treatment with intravenous acyclovir, was negative (figure).

The patient underwent a prolonged hospital course complicated by persistent fevers, bacterial pneumonia, and cardiac arrhythmias. Despite treatment with acyclovir for 10 days, her neurological state did not improve. Repeat lumbar punctures were performed on days 8 and 21 , both of which showed a persistent cerebrospinal fluid pleocytosis, and a raised protein concentration. She received another course of acyclovir because of these persistent abnormalities, but did not improve very much, and was discharged to a nursing home one month after admission.

\section{Discussion}

HSV is the most common aetiology of encephalitis in the United States, accounting for $10 \%$ of cases, with an annual incidence of 0.3 cases $/ 100000$ people. ${ }^{4} \mathrm{HSV} 1$ is responsible for almost all cases. Treatment with acyclovir significantly decreases morbidity and mortality. ${ }^{5}$ Unfortunately, the diagnosis of HSVE can not be definitively made on clinical grounds alone and may mimic other central nervous system disorders. ${ }^{6}$ Culture of cerebrospinal fluid is usually negative. Serological analysis of simultaneously drawn cerebrospinal fluid and serum samples as well as proof of intrathecally synthesised IgM may be diagnostic, but not until three to 10 days after the onset of neurological symptoms. ${ }^{7}$ Although several non-invasive techniques, such as serology, antigen detection tests, EEG and radionuclide brain scanning have been used to diagnose $\mathrm{HSV}$, encephalitis brain biopsy remains the gold standard. ${ }^{8}$ However, many doctors are reluctant to perform a brain biopsy owing to the invasive nature of the procedure.

Recent studies have shown that PCR can detect HSV DNA sequences in cerebrospinal fluid from patients with HSV encephalitis as early as 24 hours after the onset of symptoms. ${ }^{2}$ Similarly, HSV specific DNA sequences have been detected in paraffin wax embedded sections of human brain at necropsy. In our patient PCR was invaluable for the acute diagnosis of HSV infection. The case was clarified by PCR and the diagnosis of HSV encephalitis was made without performing a brain biopsy. As this technique becomes more widely available the diagnosis of HSV encephalitis should become relatively straightforward, thus allowing for rapid identification of patients who will benefit from treatment with acyclovir.

1 Eisenstein BI. The polymerase chain reaction a new method of using molecular genetics for medical diagnosis. N Engl ₹ Med 1990;322:178-83.

2 Rowley AH, Whitley RJ, Lakeman FD, Wolinsky SM. Rapid detection of herpes-simplex-virus DNA in cerebrospinal fluid of patients with herpes simplex encephalitis. Lancet 1990;335:440-1.

3 Aslanzadeh J, Osmon DR, Wilhelm MP, Espy MJ, Smith TF. A prospective study of the polymerase chain reaction for detection of herpes simplex virus in cerebrospinal fluid submitted to the clinical virology laboratory. Mol Cell Probes 1992;6:367-73.

4 Whitely RJ. Viral encephalitis. N Engl f Med 1990; 323:242-50.

5 Whitely RJ. Herpes simplex virus infections of the central nervous system. Am f Med 1988;85(Suppl 2A):61-7.

6 Whitely RJ, Cobbs CG, Alford CA, Jr, Soong S, Hirsch MS, Connor JD, et al. Diseases that mimic herpes simplex encephalitis: diagnosis, presentation, and outcome. plex encephalitis: diagnosis,

7 Elitsur $\mathrm{Y}$, Carmi R, Sarov I. HSV-specific serum/CSF antibody ratio in association with HSV serum IgM antibodies in diagnosis of herpes encephalitis in infants. Isr $\mathcal{F}$ bodies in diagnosis of herp

8 Hanley DF, Johnson RT, Whitely RJ. Yes, brain biopsy should be a prerequisite for herpes simplex encephalitis treatment. Arch Neurol 1987;44:1289-90. 